\title{
Coherent oscillations and giant edge magnetoresistance in singly connected topological insulators
}

\author{
Rui-Lin Chu, ${ }^{1}$ Jian Li, ${ }^{2}$ J. K. Jain, ${ }^{3}$ and Shun-Qing Shen ${ }^{1}$ \\ ${ }^{1}$ Department of Physics and Center for Theoretical and Computational Physics, The University of Hong Kong, \\ Pokfulam Road, Hong Kong, China \\ ${ }^{2}$ Department of Theoretical Physics, University of Geneva, CH-1211 Geneva 4, Switzerland \\ ${ }^{3}$ Department of Physics, 104 Davey Lab, Pennsylvania State University, University Park, Pennsylvania 16802, USA
}

(Received 19 July 2009; published 12 August 2009)

\begin{abstract}
A topological insulator has a pair of extended states at the edge in the bulk insulating regime. We study a geometry in which such edge states will manifest themselves in a qualitative manner through periodic oscillations in the magnetoconductance of a singly connected sample coupled to leads through narrow point contacts. Detailed calculations identify the parameters for which these oscillations are expected to be the strongest, and also show their robustness to disorder. Such oscillations can be used as a spectroscopic tool of the edge states. A large change in the device resistance at small $B$, termed giant edge magnetoresistance, can have potential for application.
\end{abstract}

DOI: 10.1103/PhysRevB.80.081102

PACS number(s): 73.23.-b, 72.25.-b, 73.43.Qt

Topological insulators differ from ordinary insulators in having a pair of extended helical edge states, which results in quantum spin Hall (QSH) effect. ${ }^{1,2}$ A predicted nonzero conductance has been measured experimentally in the "inverted"-band semiconductor $\mathrm{HgTe} / \mathrm{CdTe}$ quantum well in a band insulating region. ${ }^{3}$ More experiments are beginning to explore the edge and surface-state properties in topological insulators. $^{4-8}$ An observation of extended edge states in a way that distinguishes between edge and bulk transport in a qualitative manner would be important to establish the physics of topological insulators, and one may ask if they can also exhibit other interesting phenomena. We suggest an experimentally realizable geometry, namely, a singly connected sample with two consecutive scatterers, as shown in Fig. 1; for a system with only edge transport, an effective onedimensional ring is formed, which will lead to AharonovBohm (AB) oscillations in conductance. An observation of such oscillations in a singly connected geometry will constitute an incontrovertible evidence for edge transport. Such oscillations are analogous to similar oscillations in singly connected quantum Hall systems ${ }^{9-11}$ but with an important difference: in the present case, the oscillations occur at very small or even zero magnetic fields (in zero magnetic field the oscillations occur as a function of the gate voltage) in contrast to the quantum Hall systems for which strong magnetic fields are required for the establishment of edge states. Our results also indicate the possibility of a "giant edge magnetoresistance" (GEMR), which is insensitive to the geometry of the device and may have potential for practical application.

We study here the device shown in Fig. 1, which consists of a two-dimensional strip of a topological insulator on which two quantum point contacts (QPCs) have been patterned thorough gates (shaded regions in Fig. 1). The QPCs define a saddle-shaped confining potential, whose height can be controlled by a gate voltage. An effective disk of area $A$ $=\pi R^{2}$ ( $R$ is the radius of the disk) is formed in the center. An AB effect in the device can be expected intuitively because a topological insulator possesses a pair of independent gapless edge states of different spins moving in opposite directions, each forming an ideal one-dimensional loop around the disk.
The two edge states are independent because no backscattering is allowed at a given sample edge even in the presence of weak time-reversal invariant disorder. We note here that spin is not a good quantum number in topological insulators because of spin-orbit coupling. In the absence of a magnetic field, the actual edge states are eigenstates of the timereversal operator; their characterization as spin up and down is not precisely correct, and the word "spin" below is to be viewed more generally as the quantum number denoting the two states of a Kramers doublet.

In the remainder of the Rapid Communication we study this geometry quantitatively to ascertain the optimal parameters for the effect and also to estimate the influence of the ever present disorder. We assume a weak magnetic field $B_{\perp}$ normal to the plane. Following Ref. 10 we consider a spin-up (or spin-down) electron traveling from the left-hand side (LHS) in Fig. 1. At the LHS junction it splits into two partial waves: one is transmitted through the QPC into the disk with amplitude $t$ and the other is transmitted across the QPC with an amplitude $r$ causing a backscattering. We denote the wave-function amplitudes in the upper edge and lower edge, right after the LHS junction, by $u_{1}$ and $d_{1}$, respectively. The corresponding amplitudes in the vicinity of RHS junction are

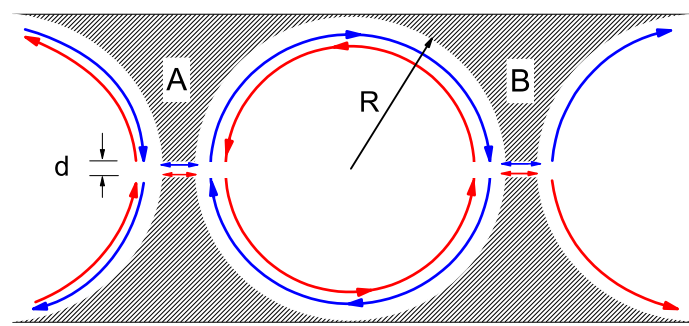

FIG. 1. (Color online) Schematic of the geometry studied in this Rapid Communication, which consists of a disk connected to two reservoirs through two quantum point contacts. Red (light gray) and blue (dark gray) lines indicate the chiral edge channels of spin-up and -down electrons, with arrows indicating the direction of their motion. In our calculations, we take the following parameters: width $W=500 \mathrm{~nm}$, length $L=1030 \mathrm{~nm}$; the slit width $d$ of the quantum point contacts is taken as a variable. 
$u_{2}=u_{1} \exp [i \theta / 2]$ and $d_{2}=d_{1} \exp [-i \theta / 2]$, where

$$
\theta=2 \pi \phi / \phi_{0}+2 \pi k R_{\text {eff }},
$$

$\phi_{0}=h / e$ is the magnetic-flux quantum, $\phi=\pi R_{\mathrm{eff}}^{2} B_{\perp}$ is the magnetic flux threading the effective one-dimensional loop with an effective radius $R_{\text {eff }}$, and $2 \pi k R_{\text {eff }}$ is the phase acquired by the wave function traveling along the loop. A partial wave goes through the RHS slit with an amplitude $t^{\prime}$ and across the slit with an amplitude $r^{\prime}$. Using the theory of multiscattering processes ${ }^{12}$ it then follows that the total transmission for spin-up electron through slits $\mathrm{A}$ and $\mathrm{B}$ is given by

$$
T^{\uparrow}\left(B_{\perp}\right)=\frac{\left|t t^{\prime}\right|^{2}}{1+\left|r r^{\prime}\right|^{2}-2\left|r r^{\prime}\right| \cos \left(\theta+\theta_{0}\right)} .
$$

Here $\theta_{0}=\arg \left(r r^{\prime}\right)$. For specificity, our numerical calculations below assume two symmetric QPCs with $|t|=\left|t^{\prime}\right|$ and $|r|$ $=\left|r^{\prime}\right|$. Resonant tunneling, i.e., $T^{\uparrow}\left(B_{\perp}\right)=1$, occurs for $\cos (\theta$ $\left.+\theta_{0}\right)=1$. The transmission coefficient for a spin-down electron $T^{\downarrow}\left(B_{\perp}\right)$, which is the time-reversal counterpart of spin-up electron at $-B_{\perp}$ field, is given by $T^{\downarrow}\left(B_{\perp}\right)=T^{\uparrow}\left(-B_{\perp}\right)$. According to the Landauer-Buttiker formula, ${ }^{13,14}$ the total conductance is $G(B)=\frac{e^{2}}{h}\left[T^{\uparrow}\left(B_{\perp}\right)+T^{\downarrow}\left(B_{\perp}\right)\right]$, which is therefore expected to be symmetric with respect to the direction of the magnetic field.

To make further progress, we consider the specific case of an "inverted"-band $\mathrm{HgTe} / \mathrm{CdTe}$ heterojunction. We consider the effective Hamiltonian for $\mathrm{HgTe} / \mathrm{CdTe}$ quantum well subject to a perpendicular field $\mathbf{B}_{\perp}, 2,15$

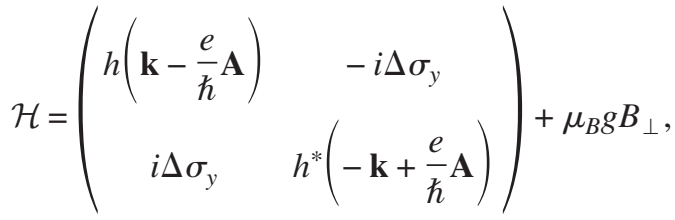

where, in the spin-up sector, $h(\mathbf{k})=-D k^{2}+A\left(k_{x} \sigma_{x}+k_{y} \sigma_{y}\right)$ $+\left(M-B k^{2}\right) \sigma_{z}$, and the wave vector $\mathbf{k}=\left(k_{x}, k_{y}\right)$ is replaced by $\mathbf{k}-\frac{e}{\hbar} \mathbf{A}$ in the presence of a magnetic field where $\mathbf{A}$ is a vector potential for magnetic field. The symbol $\sigma_{y}$ is the Pauli matrix. $A, B, D$, and $M$ are sample specific parameters, functions of the quantum well thickness; here we take $A$ $=364.5 \mathrm{meV} \mathrm{nm}^{2}, B=-686 \mathrm{meV} \mathrm{nm}^{2}, D=-512 \mathrm{meV} \mathrm{nm}^{2}$, $M=-10 \mathrm{meV}$, and $\Delta=5 \mathrm{meV}$. In the spin-down sector is the time-reversal counterpart of $h(k)$. The $\Delta$ term originates from the bulk inversion asymmetry. The $g$ factor is $g$ $=\operatorname{diag}\left(g_{E \perp}=22.7, g_{H \perp}=-1.21,-g_{E \perp},-g_{H \perp}\right)$. The combination of the bulk inversion asymmetry and the Zeeman coupling accounts for the magnetoresistance measured experimentally. ${ }^{3,15}$ To determine the conductance through the disk subjected to a perpendicular and weak field $B_{\perp}$, we use the Green's-function technique to calculate the transmission coefficients $T$ numerically in the Landauer-Büttiker formalism. ${ }^{12,16}$ For this purpose, we use a tight-binding model that reproduces the Hamiltonian in Eq. (3) in the continuum limit. In the present work, more than $200 \times 100$ lattice sites and the lattice space $a=5 \mathrm{~nm}$ were used in the calculation, and the lattice size effects are vanishingly small.

We first present the coherent oscillation of conductance in $B_{\perp}$. Figure 2 shows the conductance for several Fermi ener-

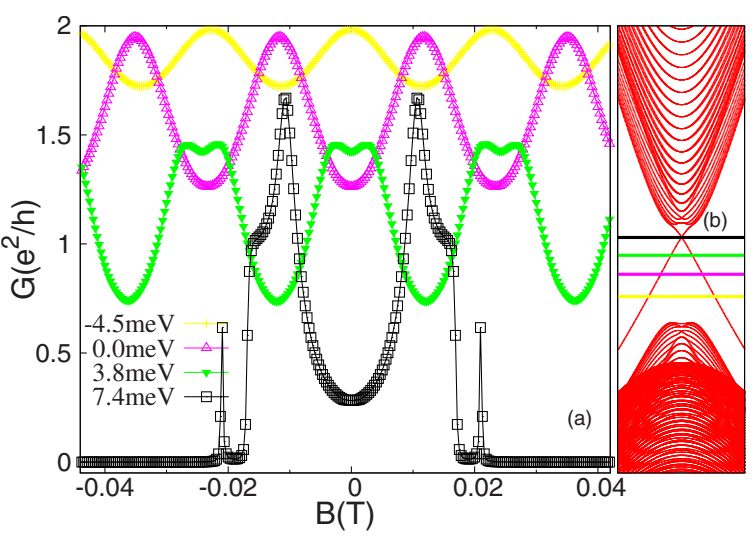

FIG. 2. (Color online) (a) Magnetoconductance $G$ for several energies in the band gap. (b) The corresponding energy locations for the curves in panel (a). Point contact constriction width $d$ $=40 \mathrm{~nm}$.

gies, whose locations in the band structure are illustrated in Fig. 2(b). The conductance exhibits periodic oscillations in $B_{\perp}$, with the period determined by the effective loop area. Upon lowering the Fermi level, the oscillation amplitude becomes smaller, but the average value of conductance becomes larger. This reflects the fact that the transmission coefficient through the two slits increases when the Fermi energy decreases. In general cases, the maximal value of the conductance does not reach $2 e^{2} / h$ because $T^{\uparrow}\left(B_{\perp}\right)$ and $T^{\downarrow}\left(B_{\perp}\right)$ do not satisfy the resonance condition simultaneously. The conductance in zero magnetic field is determined by the extra phase difference and can be either a maximal or minimal depending on the Fermi level. For the parameters adopted in this Rapid Communication, we notice an energy gap opens at $E_{f}=7.46 \mathrm{meV}$ for a nonzero magnetic field. Close to this energy, the conductance varies drastically at a small field and then rapidly drops to zero.

Figure 3 shows the dependence of oscillations on the slit width $d$. The solid lines are the best fits from Eq. (1) with three adjustable parameters $T_{0}, R_{\text {eff }}$ and $\delta \theta . T_{0}=|t|^{2}=\left|t^{\prime}\right|^{2}$ is

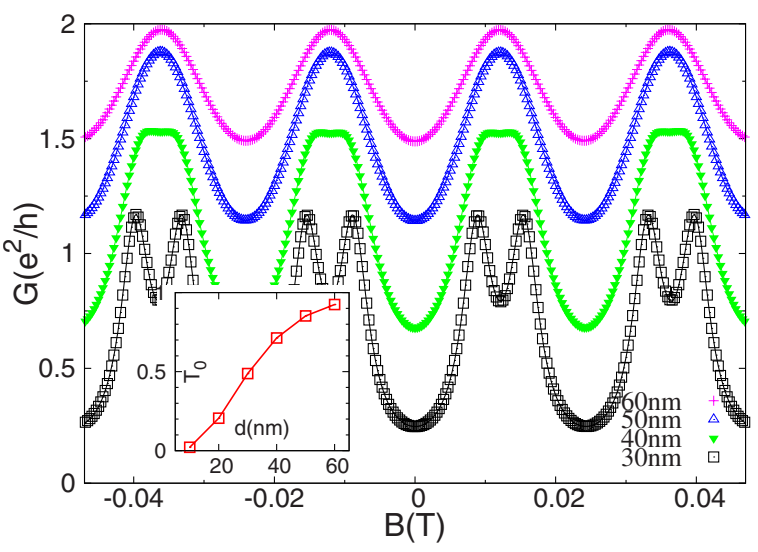

FIG. 3. (Color online) The magnetoconductance for several widths $d$ of the quantum point contacts. The solid lines are best fits using Eq. (2). The inset shows the relation between the transmission coefficient $T_{0}$ and $d$. The period is independent of $d$. All curves are evaluated for $E_{f}=4 \mathrm{meV}$. 


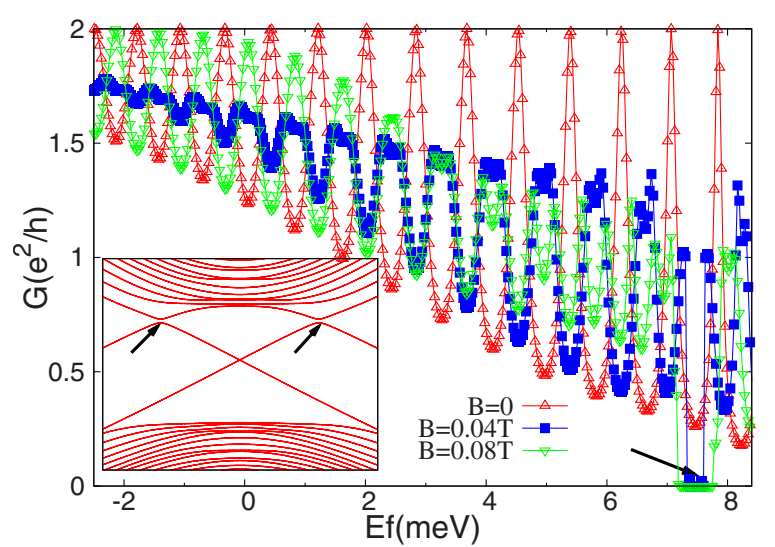

FIG. 4. (Color online) The oscillation of conductance as a function of the Fermi energy at $B_{\perp}=0,0.04$ and $0.08 \mathrm{~T}$, The period in $E_{f}$ is about $0.74 \mathrm{meV}$. Inset: the band structure at $B_{\perp}=0.08 \mathrm{~T}$. The arrows indicate the energy gap.

the transmission coefficient of electron through a single slit. The effective radius $R_{\text {eff }}=\sqrt{\phi_{0} / \pi B_{0}}$ can be deduced from the period $B_{0}$ of the oscillation; its ratio to the radius of the disk is $R_{\mathrm{eff}} / R \approx 0.95$, which reflects the finite width of an edge state near the boundary of the disk. Actually, the edge state wave function has the form ${ }^{17} \phi(y) \sim\left(e^{-y / \xi_{1}}-e^{-y / \xi_{2}}\right) /$ $\sqrt{\frac{\xi_{1}}{2}+\frac{\xi_{2}}{2}-\frac{2 \xi_{1} \xi_{2}}{\xi_{1}+\xi_{2}}}$, where $y$ stands for the distance away from the boundary, $\xi_{1} \approx 317 /(240+E)$ and $\xi_{2} \approx 317 /(13.4-E) \mathrm{nm}(E$ in units of meV) near the crossing point $k=0$ for the parameters adopted in this Rapid Communication. $\xi_{1}$ and $\xi_{2}$ are two characteristic (decay) length with typically $\xi_{1} \ll \xi_{2}$; hence the spatial distribution of the edge state is determined mainly by $\xi_{2}$. The inset in Fig. 3 shows how $T_{0}$ depends on the slit width $d$. As expected, $T_{0}$ vanishes for $d / \xi_{2} \ll 1$ and approaches unity for $d / \xi_{2} \gg 1$. Conductance oscillations are suppressed in both limits; in particular, for $d / \xi_{2} \gg 1$ it has the universal value $2 e^{2} / h$ (Refs. 1 and 2 ) and displays no magnetoresistance. The $\mathrm{AB}$ oscillations occur only when the slit width is comparable to the spatial distribution of edge state. The numerical results are in good agreement with Eq. (1) in the first few periods but exhibit an increasing deviation for larger $B$ field, which originates from the dependence of edge state spectrum on the magnetic field (we use the $B=0$ dispersion when fitting the numerical data).

A feature of this coherent effect are the rapid oscillations in the conductance as a function of the Fermi energy $E_{f}$, as shown in Fig. 4. These oscillations are caused by the phase shift $2 \pi k R_{\text {eff }}$ due to the chirality of the edge states. In the absence of bulk inversion asymmetry, the dispersion of the edge state $E(k) \approx-M D / B \pm A \sqrt{1-D^{2} / B^{2}} k \approx+7.46$ $\pm 242.5 k \mathrm{meV}$ ( $k$ in unit of $\mathrm{nm}^{-1}$ ) is linear with small $k$, and thus the phase shift can be tuned by the Fermi energy. ${ }^{17}$ The period is given by $\Delta E_{f}=A \sqrt{1-D^{2} / B^{2}} /\left(e R_{\text {eff }}\right)$. The increasing oscillation amplitude reflects that the spatial distribution of edge state is dependent on the Fermi energy. The edge state becomes wider with increasing energy, ultimately evolving into a bulk state when $E \geq 13.4 \mathrm{meV}$. Correspondingly, the transmission coefficients decreases and the oscillation amplitude increases according to Eq. (1). A measurement of such zero-field resistance oscillations will constitute direct evi-

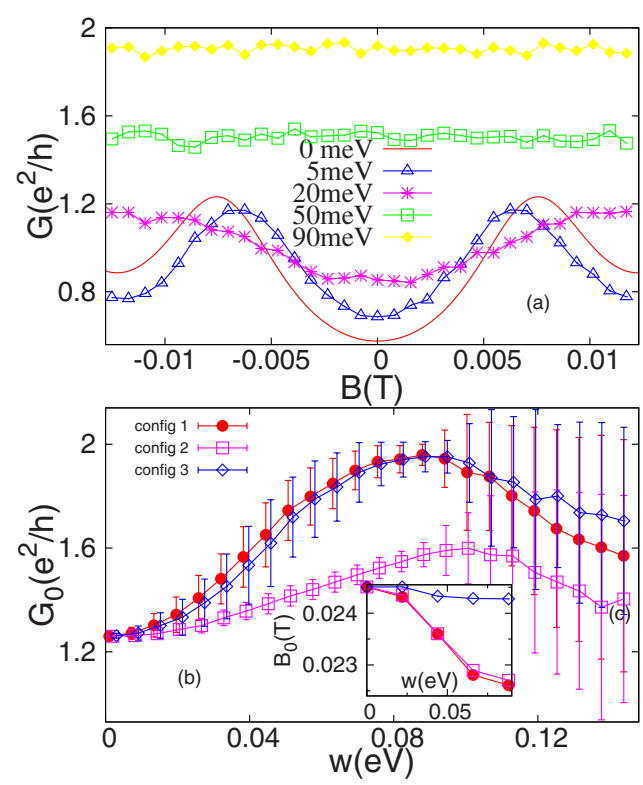

FIG. 5. (Color online) (a) Conductance oscillations as a function of disorder for several values of disorder. (b) The disorder dependence of transmission coefficient through a single QPC $T_{0}$ for three different spatial distributions of disorders. Configure 1: disorder is distributed over the entire device; 2 : no disorder in the vicinity of the QPC slit; and 3: disorder is distributed only in the vicinity of the QPC slit. The inset in (b) shows the variance in the period of magnetic field for two different spatial distribution of disorder. Averaging is performed over 200 disorder samples; $d=40 \mathrm{~nm}$ and $E$ $=5.5 \mathrm{meV}$.

dence of the existence of edge state as well as its linear dispersion in this system. For a nonzero magnetic field, an anomaly of the oscillation occurs when the Fermi level sweeps over the energy gap, and the conductance will be suppressed, which can be used to measure the energy gap.

The feasibility of the observations of the predicted magnetoresistance oscillations depends in part on how sensitive their amplitude is to the ever present disorder. Given that time-reversal invariant disorder does not cause any backscattering between two spin channels at the same edge, ${ }^{1,18,19}$ one might expect that it also does not affect the coherent magnetoresistance oscillations. We study the effect of disorder by introducing disorder as a random on-site energy with a uniform distribution within $[-w / 2, w / 2]$. The results, displayed in Fig. 5(a), demonstrate that disorder diminishes the amplitude of oscillations while enhancing the average conductance. The reduction in the coherent oscillations originates from an impurity configuration dependent phase shift $\delta \theta$ in Eq. (1); the disorder averaging over the phase shift smears the quantum interference, completely suppresses it when the variance of $\delta \theta$ is comparable with $\pi$. It is noteworthy that the $\mathrm{AB}$ oscillations survive up to a large disorder. For comparison, the hopping matrix element in Eq. (3) is $-D / a^{2} \simeq 82 \mathrm{meV}$, and numerical results indicate that it takes on-site disorder $w \geq 50 \mathrm{meV}$ to suppress the oscillations. The enhancement of the average conductance is surprising at first sight [see Fig. 5(b)], but we attribute it to a squeezing the spatial distribution of the edge states by disorder. Since the effective radius $R_{e f f}=\sqrt{\phi_{0} / \pi B_{0}}$, such squeezing can also 
be seen in the slight decrease in the oscillation period of the conductance when increasing disorder strength [see the insert in Fig. 5(b)]. The disorder around the slit plays a critical role by significantly enhancing the tunneling probability. It is because disorder closes the local energy gap (caused by the finite size of the slit) near the Fermi surface and fills up the density of states there. ${ }^{20}$ Of course, even stronger disorder leads to Anderson localization, and the edge states will eventually be destroyed, ${ }^{21-23}$ resulting in a vanishing of conductance. All our preceding discussion has assumed zero temperature. Scattering between the spin-up and spin-down edges becomes possible at nonzero temperatures due to inelastic scattering involving phonons, which is believed to cause the deviation from perfect quantization in the experiments of Ref. 3. The AB oscillations discussed above will be observable only for disks with perimeters smaller than the phase coherence length of the edge states.
In conclusion, we have studied theoretically a geometry in which the edge states of topological insulators are predicted to produce coherent oscillations in the magnetoconductance due to Aharonov-Bohm interference. This physics also produces a giant edge magnetoresistance in a weak field. If confirmed by experiments, this may be of practical interest. The edge magnetoresistance can be controlled by varying the slit width $d$ and the size of the disk. The conductance also exhibits an oscillation as a function of the Fermi energy or the gate voltage in the absence of magnetic field. The amplitude of $\mathrm{AB}$ oscillations also contains information about the transverse spatial extent of the edge channels.

This work was supported by the Research Grant Council of Hong Kong under Grants No. HKU 703708 and No. HKU 10/CRF/08.
${ }^{1}$ C. L. Kane and E. J. Mele, Phys. Rev. Lett. 95, 226801 (2005); 95, 146802 (2005).

${ }^{2}$ B. A. Bernevig, T. L. Hughes, and S. C. Zhang, Science 314, 1757 (2006).

${ }^{3}$ M. Konig, S. Wiedmann, C. Brune, A. Roth, H. Buhmann, L. W. Molenkamp, X. L. Qi, and S. C. Zhang, Science 318, 766 (2007).

${ }^{4}$ D. Hsieh, D. Qian, L. Wray, Y. Xia, Y. S. Hor, R. J. Cava, and M. Z. Hasan, Nature (London) 452, 970 (2008).

${ }^{5}$ J. W. Wells, J. H. Dil, F. Meier, J. Lobo-Checa, V. N. Petrov, J. Osterwalder, M. M. Ugeda, I. Fernandez-Torrente, J. I. Pascual, E. D. L. Rienks, M. F. Jensen, and Ph. Hofmann, Phys. Rev. Lett. 102, 096802 (2009).

${ }^{6}$ X. L. Qi, R. D. Li, J. D. Zang, and S. C. Zhang, Science 323, 1184 (2009).

${ }^{7}$ C. Y. Hou, E. A. Kim, and C. Chamon, Phys. Rev. Lett. 102, 076602 (2009).

${ }^{8}$ T. Yokoyama, Y. Tanaka, and N. Nagaosa, Phys. Rev. Lett. 102, 166801 (2009).

${ }^{9}$ J. K. Jain, Phys. Rev. Lett. 60, 2074 (1988).

${ }^{10}$ U. Sivan, Y. Imry, and C. Hartzstein, Phys. Rev. B 39, 1242 (1989).

${ }^{11}$ B. J. van Wees, L. P. Kouwenhoven, C. J. P. M. Harmans, J. G. Williamson, C. E. Timmering, M. E. I. Broekaart, C. T. Foxon, and J. J. Harris, Phys. Rev. Lett. 62, 2523 (1989).

${ }^{12} \mathrm{~S}$. Datta, Electronic Transport in Mesoscopic Systems (Cambridge, London, 1995), pp. 129 and 296-321.

${ }^{13}$ R. Landauer, Philos. Mag. 21, 863 (1970).

${ }^{14}$ M. Büttiker, Phys. Rev. B 38, 9375 (1988).

${ }^{15}$ M. König, H. Buhmann, L. W. Molenkamp, T. Hughes, C. X. Liu, X. L. Qi, and S. C. Zhang, J. Phys. Soc. Jpn. 77, 031007 (2008).

${ }^{16}$ J. Li, L. Hu, and S. Q. Shen, Phys. Rev. B 71, 241305(R) (2005); J. Li and S. Q. Shen, ibid. 76, 153302 (2007).

${ }^{17}$ B. Zhou, H. Z. Lu, R. L. Chu, S. Q. Shen, and Q. Niu, Phys. Rev. Lett. 101, 246807 (2008).

${ }^{18}$ C. Wu, B. A. Bernevig, and S. C. Zhang, Phys. Rev. Lett. 96, 106401 (2006).

${ }^{19}$ C. Xu and J. E. Moore, Phys. Rev. B 73, 045322 (2006).

${ }^{20}$ K. C. Beverly, J. L. Sample, J. F. Sampaio, F. Remacle, J. R. Heath, and R. D. Levine, Proc. Natl. Acad. Sci. U.S.A. 99, 6456 (2002).

${ }^{21}$ J. Li, R. L. Chu, J. K. Jain, and S. Q. Shen, Phys. Rev. Lett. 102, 136806 (2009).

${ }^{22}$ M. Onoda, Y. Avishai, and N. Nagaosa, Phys. Rev. Lett. 98, 076802 (2007).

${ }^{23}$ D. N. Sheng, Z. Y. Weng, L. Sheng, and F. D. M. Haldane, Phys. Rev. Lett. 97, 036808 (2006). 